\title{
CLASSIFICATION OF SIMPLE KNOTS BY BLANCHFIELD DUALITY
}

\author{
BY C. KEARTON
}

Communicated by William Browder, January 22, 1973

0 . Introduction. The purpose of this paper is to announce some results on simple knots, i.e. knots of $S^{2 q-1}$ in $S^{2 q+1}$ whose complements have the homotopy $(q-1)$-type of $S^{1}$. We state in $\S 4$ two theorems proved in the author's Ph.D. thesis [2] which give a classification of simple $(2 q-1)$ knots, $q>3$, in terms of the Blanchfield duality pairing associated with the infinite cyclic cover (see §3). These results are proved using a generalization of the presentation method for classical knots.

Any simple $(2 q-1)$-knot has associated with it an integer matrix $A$, called the Seifert matrix, such that $\left({ }^{*}\right) A+(-1)^{q} A^{\prime}$ is unimodular. $A$ is not unique, but is determined up to $S$-equivalence (see [4], [7]). Kervaire [6] and Levine [4] show that the $S$-equivalence classes of integer matrices satisfying $\left({ }^{*}\right)$ are in 1-1 correspondence with simple $(2 q-1)$-knots for $q \geqq 3$. They work in the differential category, but their proofs use handlebody techniques and are applicable to the PL category in which we work.

It is not hard to see that the modified Blanchfield pairing may be described in terms of $A$, using Seifert's construction for the infinite cyclic cover. Thus, via the geometric results, for $q>3$ the modified Blanchfield pairing determines, and is determined by, the $S$-equivalence class of the Seifert matrix. But the algebra depends only on the value of $q \bmod 2$, and so this is true for all $q$.

Trotter [7] has a purely algebraic proof that two Seifert matrices are $S$-equivalent if and only if they determine isomorphic Blanchfield pairings.

1. Preliminaries. We shall work throughout in the piecewise linear category, and it is to be understood that all submanifolds are locally flat. An $n$-knot is an oriented pair $\left(S^{n+2}, S^{n}\right)$, where $S^{r}$ denotes an $r$-sphere, and $S^{n}$ is a submanifold of $S^{n+2}$. Two $n$-knots are equivalent if there is an isomorphism of pairs between them which preserves orientations. We define $K$, the complement of the $n$-knot, to be the complement of the interior of a regular neighbourhood of $S^{n}$. An $n$-knot is $r$-simple if $K$ has

AMS (MOS) subject classifications (1970). Primary 55C05, 57C45; Secondary 55B45, $57 \mathrm{C} 10$.

Key words and phrases. $n$-knot, simple knot, Blanchfield duality pairing, knot presentation. 
the homotopy $r$-type of $S^{1}$. Following Levine [4], we call a $(q-1)$-simple $(2 q-1)$-knot simple.

It was shown in a recent paper by Kearton and Lickorish [3] that any closed submanifold $M$ of the interior of $Q \times I$ can be isotoped into critical level position, provided that the dimension of the manifold $Q$ is not less than that of $M$. A critical level embedding is one which, regarding $M$ as $M=$ handle + collar + handle + collar $+\cdots$, embeds each handle in a level of $Q \times I$ and each collar productwise along the $I$ direction.

A presentation of an $n$-knot is obtained by removing from $S^{n+2}$ the interiors of two $(n+2)$-balls, disjoint from $S^{n}$ and each other, and moving $S^{n}$ into critical level position in the $S^{n+1} \times I$ which is left. For the case $n=1$, we obtain what is essentially a presentation in the classical sense: the main difference is that we allow vertical collars. A presentation is called $r$-simple if it has only one handle of $S^{n}$ of index less than $r$ (there must always be at least one 0 -handle of $S^{n}$ ). The dual presentation is obtained by turning the given presentation upside down, i.e. by reversing the orientations of $S^{n+1}$ and $I$ in $S^{n+1} \times I$.

$\tilde{K}$ denotes the cover of $K$ corresponding to the kernel of the Hurewicz map $\pi_{1}(K) \rightarrow H_{1}(K)$. By Alexander-Poincaré duality, $H_{1}(K) \simeq(t:)$, the free group on one generator, and so $\tilde{K}$ is an infinite cyclic cover: note that the orientations provide, via the duality isomorphisms, a preferred generator $t$ of $H_{1}(K) .(t:)$ acts on $\tilde{K}$ as a group of covering transformations, and so by linearity $H_{*}(\tilde{K})$ is a $Z[t]$-module in each dimension, where $Z[t]$ denotes the integral group ring of $(t:)$.

2. Initial results. Consider a presentation of an $n$-knot. By a result of Rourke [5], we may assume that the handles of $S^{n}$ are added in order of increasing index. Each 0-handle of $S^{n}$ corresponds to a generator of $\pi_{1}(K)$, and each 1-handle to a relation: thus we obtain a representation of the fundamental group. Following Rourke [5], we may regard the relations as obstructions to cancelling the handles. If the knot is 1 -simple, then the group presentation may be reduced to $(t:)$ by certain standard moves similar to Tietze moves. If $n>3$, these moves may be induced by handle moves in the knot presentation, using the remark above, and so we obtain the following result.

THEOREM 1. If $n>3$, a 1-simple $n$-knot has a 1-simple presentation.

For the handles of higher index, we consider the presentation of $H_{*}(\tilde{K})$ as a $Z[t]$-module yielded by the presentation of the $n$-knot, to obtain:

THEOREM 2. Given an $(r-1)$-simple presentation of an $r$-simple $n$-knot, $n>3,2 r<n$, we may ambient isotope the presentation to be r-simple. 
COROLlaRY. Any $r$-simple $n$-knot, $n>3,2 r<n$, has an $r$-simple presentation of which the dual presentation is also r-simple.

3. Blanchfield duality. Let $R=Z[t]$, and let $R_{0}$ denote the field of fractions of $R$. Conjugation in $R$ is defined as the linear extension of the map $t \rightarrow t^{-1}$, and the conjugate of an element $\alpha$ is denoted by $\bar{\alpha} . R_{0} / R$ denotes the quotient of $R_{0}$ by $R$, regarded as $R$-modules.

For any $n$-knot, the work of Blanchfield [1] provides us with a duality pairing

$$
V: H_{r}(\tilde{K}, \partial \tilde{K}) \times H_{n-r+1}(\tilde{K}) \rightarrow R_{0} / R
$$

which is sesquilinear, i.e. it is linear in the first variable and conjugate linear in the second. $V$ is a pairing of $R$-modules, and is defined by means of linking and the covering space action of $(t:)$.

If $j_{*}: H_{r}(\tilde{K}) \rightarrow H_{r}(\tilde{K}, \partial \tilde{K})$ is the isomorphism of homology in the long exact sequence of the pair $(\tilde{K}, \partial \tilde{K})$, then we define

by

$$
[,]: H_{r}(\tilde{K}) \times H_{n-r+1}(\tilde{K}) \rightarrow R_{0} / R
$$

$$
[u, v]=V\left(j_{*} u, v\right) \text {. }
$$

Then $[$,$] is also sesquilinear, and is called the modified Blanchfield$ pairing.

In the next section we shall have occasion to refer to modules with the following properties.

(i) $M$ is a finitely generated $Z[t]$-torsion-module.

(ii) $J: M \rightarrow M$ is an isomorphism, where $J x=(1-t) x$.

(iii) $M$ is $Z$-torsion-free.

(iv) There is a nonsingular sesquilinear pairing, $[]:, M \times M \rightarrow$ $R_{0} / R$, where $R=Z[t]$, which satisfies $[u, v]=(-1)^{q+1}[\overline{v, u}]$ for all $u$, $v \in M$.

By nonsingular we mean that the associated map $\bar{M} \rightarrow \operatorname{Hom}\left(M, R_{0} / R\right)$ is an isomorphism.

4. Simple knots. Applying the corollary to Theorem 2, we see that any simple $(2 q-1)$-knot has a presentation with only $(q-1)$ and $q$-handles in the middle dimensions. This enables us to get to grips with the Blanchfield pairing, in terms of a matrix presentation. By means of handle moves, we prove the following results.

THEOREM 3. If $K_{1}, K_{2}$ denote the complements of two simple $(2 q-1)$ knots, $q>3$, and there is an isomorphism $h: H_{q}\left(\tilde{K}_{1}\right) \rightarrow H_{q}\left(\tilde{K}_{2}\right)$ which preserves the modified Blanchfield pairing, then the two knots are equivalent. 
THEOREM 4. The homology module $H_{q}(\tilde{K})$ and the modified Blanchfield pairing of a simple $(2 q-1)$-knot satisfy properties (i)-(iv) of \$3. Conversely, given any such module and pairing we may construct a simple $(2 q-1)-k n o t$, $q>3$, which has these as its homology and modified Blanchfield pairing.

ADDENDUM. By the remarks in $\S 0$, Levine's results [4] show that Theorem 3 is valid for $q \geqq 2$ and Theorem 4 for $q \neq 2$.

5. Acknowledgements. I should like to thank my research supervisor, Dr. W. B. R. Lickorish, for his advice and encouragement over the past three years. During this period I was supported financially by a grant from the Science Research Council.

\section{REFERENCES}

1. R. C. Blanchfield, Intersection theory of manifolds with operators with applications to knot theory, Ann. of Math. (2) 65 (1957), 340-356. MR 19, 53.

2. C. Kearton, Presentations of n-knots, Ph.D. Thesis, University of Cambridge, 1972.

3. C. Kearton and W. B. R. Lickorish, PL critical levels and collapsing, Trans. Amer. Math. Soc. 170 (1972), 415-424.

4. J. Levine, An algebraic classification of some knots of codimension two, Comment Math. Helv. 45 (1970), 185-198. MR 42 \#1133.

5. C. P. Rourke, Embedded handle theory, concordance and isotopy, Warwick University (preprint).

6. M. A. Kervaire, Les noeuds des dimensions supérieures, Bull. Soc. Math. France 93 (1965), 225-271. MR 32 \#6479.

7. H. F. Trotter, On S-equivalence of Seifert matrices, (to appear).

Department of Mathematics, Princeton University, Princeton, New Jersey 08540

Current address: Department of Mathematics, Corpus Christi College, Cambridge, England 